\title{
"I would call them, it seems faster". The state of Telemedicine in Scotland.
}

\author{
Georgios Sfakakis \\ School of Computing \\ Edinburgh Napier University \\ 40404748@live.napier.ac.uk
}

\author{
Emilia Sobolewska \\ School of Computing \\ Edinburgh Napier University \\ e.sobolewska@napier.ac.uk
}

\begin{abstract}
The novel Coronavirus pandemic has affected the way people interact with others and services. Online remote solutions have been proposed for most interactions including health care. This position paper shows that online health services are under-utilised during Covid-19, and people are often not aware of their existence. Using primary health care (General Practitioners - GPs) and the Near Me and Telecare programs in Scotland as examples, we argue that improving the usability of the GP websites can make Telemedicine more accessible and increase its adoption.
\end{abstract}

Telemedicine. Human-Computer Interaction. Covid-19. User Experience

\section{INTRODUCTION}

Telemedicine can play a key role during healthcare emergencies such as the Covid-19 pandemic. Using remote health services, people can receive treatment from the comfort and safety of their homes without being exposed to the danger of infection through physical contact with others. The spread of highly contagious diseases can be suppressed by reduced face-to-face interactions and thus provide health care systems with more time to prepare and respond to emergencies. For people with vulnerable immune systems or disabled people that are dependent on others, Telemedicine can seamlessly provide healthcare solutions. While researchers have highlighted the importance of Telemedicine and how it can benefit both patients and clinicians [1][2][3], so far little was done to investigate the adoption of Telemedicine during Covid-19 with an emphasis on the existing systems and people's attitude towards them. Telemedicine can be an important part of everyday healthcare and a valuable tool during a pandemic [4].

This study aimed to investigate to what extend Telemedicine is utilised during the Covid-19 outbreak in Scotland and to examine whether there are specific aspects of online health services that could be improved. The study focused on Telemedicine services offered by General Practitioners (GPs), along with existing Telemedicine programmes.

\section{RELATED WORK}

Health telematics is a term defined by the World Health Organization (WHO) in 1997 to describe all remote health activities or services making use of information technology to provide health education, care, disease control, and research [5]. Today, the WHO uses the term eHealth to describe all healthrelated activities facilitated by information and communication technologies (ICT).

The benefits of Telemedicine are manifold; it can help overcome geographical constraints to receive care in remote locations, whilst disabled people can obtain consultations and treatment without having to leave their homes and endure travelling difficulties [1]. Patients require less time for a virtual visit to the doctor compared to a physical visit, which substantially reduces the time taken off work or other daily routines. Telemedicine can be of use for the elderly, as the ageing population leads to a rise in cases of chronic diseases [2], or people with conditions such as anxiety who will experience greater amounts of emotional burdens during times such as the current pandemic [6]. It can be also applied to protect carers and patients from infected people [4].

There is an increased adoption of digital health services across Europe [7]. The study investigated 5,793 GPs from 27 European countries, and showed that most GPs use ICT systems to keep medical records electronically (93\%), 78\% receive laboratory reports digitally, and $66 \%$ certify sick leave remotely. The same 2019 study showed that practitioners across Europe are not utilising 
Telemedicine services such as remote consultations and remotely monitoring patients. Only $5 \%$ of 5,793 GPs reported they perform online consultations, and another 6\% reported they have the option but choose not to. Among those who were able to offer remote health monitoring, $16 \%$ decided not to use it, which is down from $26 \%$, in 2013.

Moreover, the study looked into the online services that GPs offer through their websites. The most frequent options were to request an appointment and repeat a prescription with $43 \%$ and $36 \%$ respectively. Meanwhile, fewer than $8 \%$ of practitioners stated that although they are able to offer these, they choose not to. While the adoption of those services was lower than $50 \%$, there was a $13 \%$ increase for appointment request services and $11 \%$ for repeat prescription services since 2013.

Telemedicine must be well integrated into healthcare systems to be effectively used for emergencies such as the pandemic [10]. Sporadic use by a few clinicians will not enable people to adapt to Telemedicine solutions quickly. They must be given enough motivation to employ such technologies as it appears that they are not willing to accept them mostly due to the complexity and training required [11].

Despite the advantages of Digital Health and Telemedicine, some concerns have been raised. Primarily, a data protection and privacy must be addressed [12]. By digitising health services, newly created personal information about patients now has an online presence, vulnerable to potential attacks. Health organizations must make sure that any online interactions and data are secured to avoid breaches leading to potential discrimination based on a patient's health status. Laws such as the 'Genetic Information Non-discrimination Act' in the US [13] and the 'Code on Genetic Testing and Insurance' in the UK [14] protect patients from being treated differently based on their health conditions.

The importance of understanding patient's digital health literacy levels allows recommending Telemedicine options that would suit their individual skills, rather than being exclusively tied to the nature of the health condition that needs to be treated. Among the most frequent barriers between clinicians and Telemedicine services is the technical skills required, while among patients, the most frequent barriers are age, level of education, and technical literacy [15]. It is important that while making digital systems available to people, means of improving their digital skills as well as educating themselves on health-related matters must be also provided [16]. A survey conducted in England, found that health literacy is closely connected with the use of the Internet [16]. More specifically, it appears that people with higher levels of health literacy are more likely to use the Internet for health-related purposes.

Similar to any other physical or digital service, Telemedicine solutions must offer high-quality services, and undergo a standard way for validating the quality according to its Technical, Clinical, and System aspects [17]. For instance, with the rising use of sensors in Telemedicine, it needs to ensure adequate quality and accuracy of results, to be able to correctly diagnose any conditions and avoid false health insights [18].

\subsection{Telemedicine in Scotland}

In 2014, the Scottish Government created the Technology Enabled Care (TEC) platform (tec.scot), to promote digital health services across the country. TEC aims to provide remote access to healthcare services, to improve people's health and well-being.

Along with the Scottish Government and local health communities, TEC has formed the Digital Telecare programme (telecare.digital.office.scot), to assist disabled people, those with health conditions, or vulnerable individuals [19]. A person subscribed for Telecare receives a technology package to monitor various conditions around the house (e.g., Fire alarms, flood and movement detectors, pill reminders, etc.). In emergencies, a designated Alarm Receiving Centre (ARC) collects calls and acts accordingly to the situation. ARC might reach out to an emergency contact of the patient, a GP, or call for an ambulance. According to the Digital Office [19], there are 128,750 people receiving telecare services across Scotland with majority $(71 \%)$ being over the age of 75 .

In 2018, BT announced that by 2025 it aims to replace all analogue telephone networks with digital internet protocol services (IP) [20]. The Digital Telecare aims to research the impact and benefits of this change to telemedicine and to establish a roadmap to gradually change all analogue telecare services (telephony connection-based) to digital services (IP-based) to be able to keep up with the changes in technology and offer high-quality telecare services.

An important part of TEC is the "Near Me" program (nearme.scot), allowing patients and healthcare professionals to establish a video conference session, used by NHS Scotland for remote consultations. Such a service facilitates disabled people or people vulnerable to infections to limit their physical visits to the doctor only when necessary. Also, it helps to contain the spread of infectious diseases [21]. Furthermore, it is superior to phone consultations as it adds visual stimuli where the practitioner can understand when a patient is not feeling comfortable or is having trouble understanding the conversation. The only 
identified drawback of $\mathrm{Near} \mathrm{Me}$ is that through video conferencing sessions the level of stress and anxiety of a patient cannot be observed as well as during in-person encounters.

Telemedicine is an important developing part of the healthcare system and can be a useful tool benefiting healthcare provides as well as patients. For instance, vulnerable and disabled people could gain substantial help through Telemedicine services [22]. We aim to investigate if people are aware that such services exist, how/whether they interact with them, and to what extent the relationship with these systems was affected by the system design.

\section{METHOD}

This study used a mix of qualitative and quantitative methods; investigation took place online to abide by social distancing rules.

The questionnaire, to gather data regarding remote care was implemented in Google Forms (https://forms.gle/9QtQgUzAuwywZu5g8) and included 32 questions, divided into 4 parts: users' relationships with technology; awareness of Telemedicine in Scotland; the frequency and purpose of using GP's websites, and how the Coronavirus impacted that interaction. Demographic data was collected alongside the survey.

Questionnaire was shared online through social media platforms (July 2020) targeting local Scottish communities. All participants provided consent.

55 people took part in the survey, with all responses being fully completed and valid.

The content analysis was conducted to map current online resources offered by GPs, to identify services they offer, and to investigate promotion (or lack of thereof) of TEC programmes. It included 10 central GP Surgeries from the four biggest cities in Scotland, three each from Glasgow and Edinburgh, and two each from Aberdeen and Dundee.

The systems were subsequently evaluated in an online session by User Experience practitioners. Three subject experts ( 2 females, 1 male, aged 2530) were asked to find basic information about the surgery (contact information, opening hours), book an appointment, and receive test results, whilst employing the think-aloud method to externalise their thoughts. They also evaluated the usability and accessibility of the systems using a SUS questionnaire [23], 10 Usability Heuristics for User Interface Design [24], as well as accessibility guidelines [25].

\section{RESULTS}

The questionnaire was returned by 55 participants: 22 Females, 32 Males, and one Nonbinary person. The majority of the participants were between the ages of 18 and $39(n=48)$, followed by seven people aged 40-69. 25 participants obtained master's degree, 17 Bachelor's, whilst the rest $(n=13)$ completed studies at a Higher National Degree or lower. Most participants $(n=32)$ were employed full-time, 14 were students, four were part-time employed, three self-employed and two retired or furloughed. Male participants were mostly represented in the younger age groups of 18-29 $(n=16)$ followed by 30-39 $(n=14)$. Female participants had a more evenly spread representation across all age groups with the largest one being the 30-39 $(n=9)$.

Only 16 males and eight females were familiar with the term Telemedicine. Three female and five male participants stated that they are aware of the Telecare program. The $76 \%$ of the participants have not heard of Telemedicine before.

Concerning the Near Me program, only four males and six females were aware of it. The $80 \%$ of the participants are not aware of the service.

The majority of the participants (89\%) stated that health care can benefit from Telemedicine. When the participants were asked if Telemedicine can benefit them, $(60 \%)$ agreed or strongly agreed, $34 \%$ were undecided. The majority of the undecided were Males $(n=13)$ with most of them $(n=8)$ aged $18-29$. Only six females stated that they were undecided.

Regarding online resources, before and during the pandemic, 22 people were instructed by their GP to engage with their website, with the majority of them $(n=15)$ following through.

The content analysis focussed on identifying the most popular online services offered by GPs. All surgeries extended the option to repeat a prescription online, and to book an appointment, as reported by the European Commission's study [7]. Half of the websites $(n=5)$ mentioned the option to attend a video consultation with a doctor or a nurse while three had the option to register patients online. Out of the 10, only two practices had a mention of the Telecare program.

The usability testing of the websites [24][25] concluded that most common issues include distracting pop-ups $(n=4)$, and text-based pages $(n=6)$ that were uneasy to search for relevant content. The expert evaluation with UX professionals shown, that though they were able to complete the tasks, they found the systems to be difficult to use, with many inconsistencies and 
unnecessary complexity. They have reported that pages have issues with unclear structure and navigation; they are overloaded making it difficult to browse through and find information; the lack of consistency including different colours, fonts, and font sizes used across pages, confuse the user. Furthermore, different surgeries did not share common guidelines to achieve consistency across systems.

Lastly, the experts evaluated the usability of the systems with the SUS questionnaire. The average score for the system was 34 with 68 being the minimum recommended [23].

\section{RE-DESIGN AND EVALUATION}

Based on the data analysis and the expert evaluation results, an alternative design was implemented, tested, and evaluated using usercentred, iterative approach.

The process included gathering requirements to define concrete scenarios, as well as prototyping and testing of initial designs. Subsequently, new design was created according to relevant findings; a high-fidelity wireframe was produced to evaluate final outcome.

The proposed design was built to facilitate an accessible system. Pale colours in darker shades of blue were used for the design to be pleasant to the eye. The blue was also picked as it is the main colour of the NHS. Black text on a white background was used to display important information while descriptive images and icons were incorporated. The font selected was Arial with $12 \mathrm{pt}-14 \mathrm{pt}$ for main text and 18pt - 22pt for headlines and titles. To test the design language, a high-fidelity wireframe was created.

The prototype was tested and evaluated by 13 participants, 10 males and three females 18-39 years old, using a modified version of the Microsoft Reaction Card Method [28]. The aim was to replicate a set of frequent scenarios and tasks to test its usability and gather user feedback.

The testing session consisted of three tasks-based scenarios, including finding information about the surgery, booking an appointment, repeating a prescription, and verifying their registered requests. Prototype was fully functional.

Initially, the participants were asked to describe the system with two words (e.g., colourful, old). Most word choices suggested appropriate design, with answers such as 'professional', 'simple', classic', and 'medical' being quoted. Subsequently, participants were given a list of 20 words from the Microsoft Desirability Toolkit [28] and were prompted to pick three that best describe the system. The most popular choice was
'Understandable' and 'Consistent' two important characteristics for such a system.

Importantly, the new design improved interactions with online health services through better usability. When reviewed by experts, the proposed design achieved 88 points on the system usability scale while the average score for the existing systems that were evaluated by the experts was 34 points. This suggests that existing resources have significant space for improvements.

\section{DISCUSSION AND CONCLUSIONS}

Limited knowledge of existing Telemedicine services has been one of the reasons keeping its adoption at low levels [29][30], which as our study shows, is also true to Scotland. Near Me and Telecare, two major parts of Scotland's effort to offer technology-enabled health care have little or no recognition with $18.2 \%$ and $14.5 \%$ respondents respectively reporting to have heard of them. Meanwhile, more than $60 \%$ admitted that they know or might know someone who could benefit from such service. The low awareness and adoption of resources can be correlated with the frequency it is mentioned in primary care websites - only two out of the ten analysed resources referred to Telecare. Instead, the overwhelming majority of respondents reported using generic Internet content to search for health-related information (92.7\%).

Despite participants having little to no knowledge of Telemedicine prior to our study, respondents have shown a positive attitude towards it, with $89 \%$ of them stating that it can benefit health care while $60 \%$ reporting that they could personally benefit from it. It appears that male participants aged 1829 years old are the ones that admit knowing most about such systems while remaining undecided towards them. The female participants have shown a better response regarding the actual use of Telemedicine regardless of their age, which also corresponds with the numbers of Telemedicine users previously reported [8].

Based on the analysis and the experts' evaluation, most GP's websites have major usability issues. While some offer online services, weaknesses in information architecture, quality and quantity of content, navigation, consistency, and design, make systems difficult to use. A simple re-design to address those issues has shown a significand improvement in usability and information retrieval. After modifications, the SUS [23] score improved from 34 to $88 / 100$. A common design language could be used to ensure consistency across different websites. Lastly, creating more accessible and usable resources could create better exposure to Telemedicine, and in turn increase adoption. 


\section{REFERENCES}

[1] Kahn, J. M. (2015). Virtual Visits Confronting the Challenges of Telemedicine. The New England Journal of Medicine, 372(18), 1684-1685.

[2] Van den Berg, Neeltje, Schumann, Maika, Kraft, Kathleen, and Hoffmann, Wolfgang. (2012). Telemedicine and telecare for older patients-A systematic review. Maturitas, 73(2), 94-114.

[3] Bashshur, R., Doarn, C. R., Frenk, J. M., Kvedar, J. C., and Woolliscroft, J. O. (2020). Telemedicine and the COVID-19 Pandemic, Lessons for the Future.

[4] Hollander, J. E., and Carr, B. G. (2020). Virtually perfect? Telemedicine for COVID-19. New England Journal of Medicine, 382(18), 1679-1681.

[5] WHO Group Consultation on Health Telematics (1997: Geneva, Switzerland). (1998). A health telematics policy in support of WHO's Health-for-all strategy for global health development: report of the WHO Group Consultation on Health Telematics, 11-16 December, Geneva, 1997. World Health Organization.

https://apps.who.int/iris/handle/10665/63857 (Accessed July, 2020)

[6] Zhou, X., Snoswell, C. L., Harding, L. E., Bambling, M., Edirippulige, S., Bai, X., and Smith, A. C. (2020). The role of telehealth in reducing the mental health burden from COVID-19. Telemedicine and e-Health, 26(4), 377-379.

[7] Lupiáñez-Villanueva, F., Devaux, A., Valverde-Albacete, J., Folkvord, F., Fauli, C., Altenhofer, M., ... and Harshfield, A. (2019). Benchmarking deployment of eHealth among general practitioners.

[8] Barnett, M. L., Ray, K. N., Souza, J., and Mehrotra, A. (2018). Trends in telemedicine use in a large commercially insured population, 2005-2017. Jama, 320(20), 21472149.

[9] Haluza, D., Naszay, M., Stockinger, A., and Jungwirth, D. (2017). Digital natives versus digital immigrants: influence of online health information seeking on the doctor-patient relationship. Health communication, 32(11), 1342-1349.

[10] Smith, A. C., Thomas, E., Snoswell, C. L., Haydon, H., Mehrotra, A., Clemensen, J., and Caffery, L. J. (2020). Telehealth for global emergencies: Implications for coronavirus disease 2019 (COVID-19). Journal of telemedicine

and

telecare, $1357633 \times 20916567$.

[11] Wade, Victoria A, Eliott, Jaklin A, and Hiller, Janet E. (2014). Clinician Acceptance is the Key Factor for Sustainable Telehealth Services. Qualitative Health Research, 24(5), 682-694.

[12] Meskó, Drobni, Bényei, Gergely, and Győrffy (2017)

[13] National Human Genome Research Institute $\mathrm{NIH}$. (2018). Genetic Information Nondiscrimination Act of 2008. https://www.genome.gov/aboutgenomics/policy-issues/GeneticDiscrimination\#gina (Accessed September, 2020)

[14] Department of Health and Social Care. (2008). Code on genetic testing and insurance. https://www.gov.uk/government/publications/c ode-on-genetic-testing-and-insurance (Accessed April, 2021)

[15] Scott Kruse, C., Karem, P., Shifflett, K., Vegi, L., Ravi, K., and Brooks, M. (2018). Evaluating barriers to adopting telemedicine worldwide: $A$ systematic review. Journal of telemedicine and telecare, 24(1), 4-12.

[16] Estacio, E. V., Whittle, R., and Protheroe, J. (2019). The digital divide: examining sociodemographic factors associated with health literacy, access and use of internet to seek health information. Journal of health psychology, 24(12), 1668-1675.

[17] Mathews, S. C., McShea, M. J., Hanley, C. L., Ravitz, A., Labrique, A. B., and Cohen, A. B. (2019). Digital health: a path to validation. NPJ digital medicine, 2(1), 1-9.

[18] Shift, D. (2015, September). Technology tipping points and societal impact. In World Economic Forum Survey Report, available at: http://www3. weforum. org/docs/WEF_GAC15_Technological_Tippin g_Points_report_2015. pdf (Accessed 20.08. 2018).

[19] Digital Office Scotland. (2017). Digital Telecare. https://www.digitaloffice.scot (Accessed May, 2020)

[20] British Telecom. (2018). We are updating our home network.

[21]NHS Near Me. (15, May 2020). Using Near Me paediatrics. [video] https://www.youtube.com/watch?v=2RxRKGxL DaU (Accessed July, 2020)

[22] Van der Vaart, R., and Drossaert, C. (2017). Development of the digital health literacy instrument: measuring a broad spectrum of 
health 1.0 and health 2.0 skills. Journal of medical Internet research, 19(1), e27.

[23]Brooke, J. (1996). SUS: a "Quick and dirty usability. Usability evaluation in industry, 189.

[24] Nielsen, J. (2014). Usability heuristics for user interface design.

[25]W3C. (2008) Web Content Accessibility Guidelines (WCAG) 2.0. https://www.w3.org/TR/2008/REC-WCAG2020081211 (Accessed May, 2020)

[26] Friedman, Mark G, and Bryen, Diane Nelson. (2008). Web accessibility design recommendations for people with cognitive disabilities. Technology and Disability, 19(4), 205-212.

[27]Hodes, R. J., and Lindberg, D. A. (2002). Making your website senior friendly. National Institute on Aging and the National Library of Medicine.

[28] Benedek, J., and Miner, T. (2002). Measuring Desirability: New methods for evaluating desirability in a usability lab setting. Proceedings of Usability Professionals Association, 2003(8-12), 57

[29] Portnoy, J., Waller, M., and Elliott, T. (2020). Telemedicine in the Era of COVID-19. The Journal of Allergy and Clinical Immunology: In Practice, 8(5), 1489-1491.

[30] Ohannessian, R., Duong, T. A., and Odone, A. (2020). Global telemedicine implementation and integration within health systems to fight the COVID-19 pandemic: a call to action. JMIR public health and surveillance, 6(2), e18810. 